\title{
Unrelated donor versus matched sibling donor in adults with acute myeloid leukemia in first relapse: an ALWP-EBMT study
}

Annalisa Ruggeri ${ }^{1 *}$, Giorgia Battipaglia ${ }^{1,2+}$, Myriam Labopin', Gerhard Ehninger ${ }^{3}$, Dietrich Beelen ${ }^{4}$, Johanna Tischer ${ }^{5}$, Arnold Ganser ${ }^{6}$, Rainer Schwerdtfeger ${ }^{7}$, Bertram Glass ${ }^{8}$, Jurgen Finke ${ }^{9}$, Mauricette Michallet ${ }^{10}$, Matthias Stelljes ${ }^{11}$, Pavel Jindra ${ }^{12}$, Renate Arnold ${ }^{13}$, Nicolaus Kröger ${ }^{14}$, Mohamad Mohty ${ }^{1,15,16}$ and Arnon Nagler ${ }^{16,17,18}$

\begin{abstract}
Background: Allogeneic stem cell transplantation is the only curative option for patients with acute myeloid leukemia (AML) experiencing relapse. Either matched sibling donor (MSD) or unrelated donor (UD) is indicated.

Methods: We analyzed 1554 adults with AML transplanted from MSD $(n=961)$ or UD ( $n=593$, HLA-matched 10/10, $n=481 ; 9 / 10, n=112$ ). Compared to MSD, UD recipients were older (49 vs 52 years, $p=0.001$ ), transplanted more recently (2009 vs 2006, $p=0.001$ ), and with a longer interval to transplant (10 vs 9 months, $p=0.001$ ). Conditioning regimen was more frequently myeloablative for patients transplanted with a MSD (61 vs $46 \%, p=0.001$ ). Median follow-up was 28 (range 3-157) months.

Results: Cumulative incidence (CI) of neutrophil engraftment $(p=0.07)$, grades $\|-I V$ acute GVHD $(p=0.11)$, chronic GVHD $(p=0.9)$, and non-relapse mortality (NRM, $p=0.24$ ) was not different according to the type of donor. At 2 years, $\mathrm{Cl}$ of relapse (relapse incidence (RI)) was 57 vs $49 \%(p=0.001)$. Leukemia-free survival (LFS) at 2 years was 21 vs $26 \%$ ( $p=0.001)$, and overall survival (OS) was 26 vs $33 \%(p=0.004)$ for MSD vs UD, respectively. Chronic GVHD as time-dependent variable was associated with lower RI (HR 0.78, $p=0.05)$, higher NRM (HR 1.71, $p=0.001$ ), and higher OS (HR 0.69, $p=0.001)$. According to HLA match, RI was 57 vs 50 vs $45 \%,(p=0.001)$ NRM was 23 vs 23 vs $29 \%$ $(p=0.26)$, and LFS at 2 years was 21 vs 27 vs $25 \%$ ( $p=0.003$ ) for MSD, 10/10, and 9/10 UD, respectively. In multivariate analysis adjusted for differences between the two groups, UD was associated with lower RI (HR 0.76, $p=0.001)$ and higher LFS (HR 0.83, $p=0.001$ ) compared to MSD. Interval between diagnosis and transplant was the other factor associated with better outcomes (RI (HR 0.62, $p<0.001)$ and LFS (HR 0.67, $p<0.001)$ ).
\end{abstract}

Conclusions: Transplantation using UD was associated with better LFS and lower RI compared to MSD for high-risk patients with AML transplanted in first relapse.

Keywords: Acute myeloid leukemia, Relapse, Matched sibling donor, Unrelated donor

\footnotetext{
* Correspondence: annalisaruggeri80@hotmail.com

${ }^{\dagger}$ Equal contributors

${ }^{1}$ Service d'Hématologie et Thérapie Cellulaire, Hôpital Saint Antoine, AP-HP,

184 Rue du Faubourg Saint Antoine, 75012 Paris, France

Full list of author information is available at the end of the article
} 


\section{Background}

Although the outcome of patients diagnosed with acute myeloid leukemia (AML) has improved, disease recurrence remains the leading cause of treatment failure, with only a minority of patients who durably benefit from current reinduction therapies. For patients achieving complete remission (CR) after salvage chemotherapy, the overall relapse risk is nearly $45-50 \%$, but this is highly variable and is primarily determined by the biology of the disease [1]. For patients with AML, allogeneic hematopoietic stem cell transplantation (HSCT) is a curative option. Recent improvements in donor availability, conditioning regimens, and supportive care broadened the applicability of HSCT in nearly all patients with AML with better long-term outcomes. Moreover, the use of post-transplant immunomodulating strategies allowed long-term survival and better disease control.

Both myeloablative (MAC) and reduced-intensity (RIC) conditioning regimens are suitable options for treating these patients allowing disease control. In the MAC setting, regimens containing either busulfan or total body irradiation (TBI) are effective for refractory AML with a reported 2-year leukemia-free survival (LFS) of less than $30 \%$ with both regimens [2]. On the other hand, the introduction of RIC made HSCT feasible even in elderly or in young patients with significant comorbidities, not eligible for standard MAC [3]. However, post-transplant relapse remains a challenge in RIC, especially in high-risk patients with AML in primary relapse.

In this setting, donor search might be started as soon as possible. For patients lacking a full-matched sibling donor (MSD), 35-40 \% may have an unrelated donor (UD) available in donor registries [4]. Prior studies reported comparable results of HSCT from MSD versus UD [5]; however, up to date, no studies investigated the outcomes of patients transplanted from either a MSD or UD for AML in first relapse.

Understanding the impact of donor source in patients with active disease represents an area of active investigation and might be critical in the therapeutic decisionmaking process. With this background, we analyzed patients with AML in first relapse reported to the European Society for Blood and Marrow Transplantation (EBMT) registry from 2000 to 2012.

\section{Methods}

\section{Study design and definition}

We retrospectively analyzed adult patients ( $\geq 18$ years) diagnosed with AML who underwent their first allogeneic HSCT in primary relapse between 2000 and 2012, using either a MSD or matched 10/10 or mismatched 9/10 UD. Patients transplanted using cord blood or haploidentical donors were excluded to homogenously compare adult full-matched sibling or unrelated donors. The EBMT registry is a voluntary working group of more than 500 transplant centers, mostly located in Europe, that are required to report all consecutive HSCT and follow-up data once a year. Audits are routinely undertaken to establish the accuracy of the data.

Karyotype abnormalities were classified as favorable, intermediate or high risk as previously described [6]. Conditioning regimen was MAC in case of administration of total body irradiation (TBI) at a dose greater than 6 Gray (Gy), oral busulfan greater than $8 \mathrm{mg} / \mathrm{kg}$, or intravenous busulfan greater than $6.4 \mathrm{mg} / \mathrm{kg}$. All patients provided informed consent for transplant according to the declaration of Helsinki. The Review Board of the EBMT approved this study.

\section{Definitions and end points of the study}

The primary endpoint of the study was LFS according to the type of donor (MSD vs UD). Secondary endpoints included overall survival (OS), neutrophil engraftment, acute and chronic graft versus host disease (GVHD), nonrelapse mortality (NRM), and relapse incidence (RI). LFS was calculated from the date of transplant until relapse or last disease-free follow-up. Neutrophil engraftment was defined as achieving absolute neutrophil count $\geq 0.5 \times 10^{9}$ / 1 for three consecutive days. The diagnosis and grading of acute and chronic GVHD was performed using the standard criteria [7]. Relapse and death from any cause were considered events. NRM was defined as death without prior relapse.

\section{Statistical analyses}

Median values and ranges were used for continuous variables and percentages for categorical variables. Patient-, disease-, and transplant-related variables of the groups were compared using Chi-square or Fischer exact test for categorical variables, and Mann-Whitney test for continuous variables. The probabilities of OS and LFS were calculated using the Kaplan-Meier [8] method and the log-rank test for univariate comparisons.

Neutrophil engraftment, grades II-IV acute and chronic GVHD, relapse, and NRM were calculated by using the cumulative incidence (CI) estimator to accommodate competing risks. For NRM, relapse was the competing risk, and for relapse, the competing risk was NRM.

Multivariate analyses adjusted for differences between the groups were performed using Cox proportional hazards regression model for LFS and OS, and Fine and Gray's [9] proportional hazards regression model for engraftment, GVHD, NRM, and relapse. Chronic GVHD was analyzed as time-dependent factor using Cox model for LFS and OS. $p$ values were two-sided. Statistical analyses were performed with the SPSS 19 (SPSS Inc./IBM, Armonk, NY, USA) and R 3.0 (R Development Core Team, Vienna, Austria) software packages. 


\section{Results}

\section{Patient and transplant characteristics}

Baseline characteristics are summarized in Table 1. Briefly, 1554 patients fulfilling the inclusion criteria were identified; of these, 961 were transplanted with a MSD and 481 with an UD (HLA-matched 10/10, $n=481,9 / 10, n=112$ ). There were some differences between the two groups: UD recipients were older (52 vs 49 years; $p<0.001$ ) and they underwent HSCT more recently (2006 vs 2009 for UD recipients; $p<0.001)$ compared to MSD recipients. Time from diagnosis to HSCT was longer for UD recipients (10 vs 8 months, $p<0.001)$. A slightly higher proportion of secondary AML was observed in the MSD group (125 vs 113 in the UD group, $p<0.003)$.

The majority of the patients underwent a MAC regimen, especially in the MSD group (60 vs $46 \%$ for the UD group, $p<0.001)$; 186 patients received a combination of a short intensive course of chemotherapy, followed by a RIC regimen (FLAMSA-regimen) as previously described [10], including 125 TBI-containing and 66 busulfan-containing regimen. In vivo $\mathrm{T}$ cell depletion was more frequently used in UD group (99 vs $75 \%, p<0.001$ ). Ninety percent of patients in both groups $(p=0.14)$ received peripheral blood cells (PBSC) as stem cell source.

\section{Engraftment, acute, and chronic GVHD}

The CI of neutrophil engraftment was 93 vs $92 \%$ in MSD and UD recipients, respectively $(p=0.07)$. The median time for neutrophil engraftment was of 15 days (range 6-90), with no difference between the groups. Graft failure occurred in nearly $5 \%$ of patients in each group $(p=0.73)$.

According to the type of donor CI of day-100 grades IIIV acute GVHD was 26 vs $30 \%(p=0.11)$, in MSD and $\mathrm{UD}$, respectively. There was no difference in the CI of chronic GVHD at 2 years in the 2 groups $(25 \%, p=0.90)$.

\section{Relapse and NRM}

Within 100 days after HSCT, $72 \%(n=403)$ of UD recipients achieved CR, versus $66 \%(n=586)$ in MSD group $(p=0.02)$.

At 2 years, CI of relapse was higher in MSD recipients (57 vs $49 \%$ in UD recipients, $p<0.001$ ), while NRM was similar in the two groups ( $23 \%$ in MSD vs $24 \%$ in UD, $p=0.24$ ) (Fig. 1a, b).

In multivariate analysis (Table 2), patients transplanted with UD ( $\mathrm{HR}=0.76,95 \%$ CI $0.64-0.89 ; p<0.001)$, and those with a longer interval between diagnosis and HSCT $(\mathrm{HR}=0.62,95 \%$ CI 0.53-0.72; $p<0.001)$ showed lower relapse. RIC regimen was independently associated with higher relapse $(\mathrm{HR}=1.21,95 \% \mathrm{CI} 1.02-1.42 ; p<0.03)$. Also, chronic GVHD as time-dependent variable was independently associated with a lower $\mathrm{RI}(\mathrm{HR}=0.78,95 \% \mathrm{CI}$ $0.61-1.00 ; p<0.05)$ and a higher NRM $(\mathrm{HR}=1.71,95 \% \mathrm{CI}$
Table 1 Patient, disease, and transplant characteristics

\begin{tabular}{|c|c|c|c|}
\hline Characteristic (\%) & $\operatorname{MSD}(n=961)$ & UD $(n=593)$ & $p$ value \\
\hline Median follow-up, months & $30(1-157)$ & $27(1-137)$ & 0.28 \\
\hline Median age, years (range) & $49(18-75)$ & $52(18-77)$ & $<0.01$ \\
\hline Median year of HSCT (range) & $2006(2000-2012)$ & 2009 (2000-2012) & $<0.01$ \\
\hline $\begin{array}{l}\text { Interval from diagnosis } \\
\text { to HSCT, months (range) }\end{array}$ & $8.6(2-155)$ & $10.3(2.5-186)$ & $<0.01$ \\
\hline De novo AML & $836(87)$ & $480(81)$ & $<0.01$ \\
\hline SAML & $125(13)$ & $113(19)$ & \\
\hline Prior auto-HSCT & $57(6)$ & $41(7)$ & 0.59 \\
\hline \multicolumn{4}{|l|}{ Donor's gender } \\
\hline Male & $500(52)$ & $417(72)$ & $<0.01$ \\
\hline Female & $452(48)$ & $162(28)$ & \\
\hline CMV positive serostatus & & & $<0.01$ \\
\hline Patient & $472(69)$ & $210(37)$ & \\
\hline Donor & $417(62)$ & $244(42)$ & \\
\hline \multicolumn{4}{|l|}{ Conditioning regimen } \\
\hline MAC & $559(60)$ & $269(46)$ & $<0.01$ \\
\hline $\mathrm{RIC}$ & $365(40)$ & $316(54)$ & \\
\hline \multicolumn{4}{|l|}{ Conditioning details } \\
\hline BuCy & $155(16)$ & $81(14)$ & $<0.01$ \\
\hline BuFlu & $135(14)$ & $112(19)$ & \\
\hline FluMel & $89(9)$ & $81(14)$ & \\
\hline Threo-based & $36(4)$ & $26(4)$ & \\
\hline MAC TBI & $178(1)$ & $79(13)$ & \\
\hline $\mathrm{RIC} \mathrm{TBI}$ & $32(3)$ & $13(2)$ & \\
\hline Other & $336(35)$ & $201(34)$ & \\
\hline \multicolumn{4}{|l|}{ Stem cell source } \\
\hline $\mathrm{BM}$ & $107(11)$ & $60(10)$ & 0.14 \\
\hline PBSC & $854(89)$ & $533(90)$ & \\
\hline \multicolumn{4}{|l|}{ GVHD prophylaxis } \\
\hline CsA alone & $115(12)$ & $81(14)$ & $<0.01$ \\
\hline $\mathrm{Cs} A+\mathrm{MMF}$ & $182(19)$ & $187(31)$ & \\
\hline CsA + MTX & $362(38)$ & $246(41)$ & \\
\hline Other & $68(7)$ & $75(13)$ & \\
\hline Missing & $234(24)$ & $4(1)$ & \\
\hline In vivo TCD & & & $<0.01$ \\
\hline ATG & $167(17)$ & $388(65)$ & \\
\hline Alemtuzumab & $20(2)$ & $47(8)$ & \\
\hline No & $521(54)$ & $154(26)$ & \\
\hline Missing & $235(24)$ & $4(1)$ & \\
\hline
\end{tabular}

Abbreviations: MSD matched sibling donor, UD unrelated donor, HSCT hematopoietic stem cell transplantation, $s A M L$ secondary acute myeloid leukemia, auto-HSCT autologous stem cell transplantation, CMV cytomegalovirus, $M A C$ myeloablative conditioning regimen, $R / C$ reduced-intensity conditioning regimen, $B u$ busulfan, $C y$ cyclophosphamide, Flu fludarabine, Mel melphalan, Threo threosulfan, $T B I$ total body irradiation, $B M$ bone marrow, $P B S C$ peripheral blood stem cells, CSA cyclosporine A, MMF mycophenolate mophetil, MTX methotrexate, TCD T-cell depletion, ATG antithymocyte globulin 


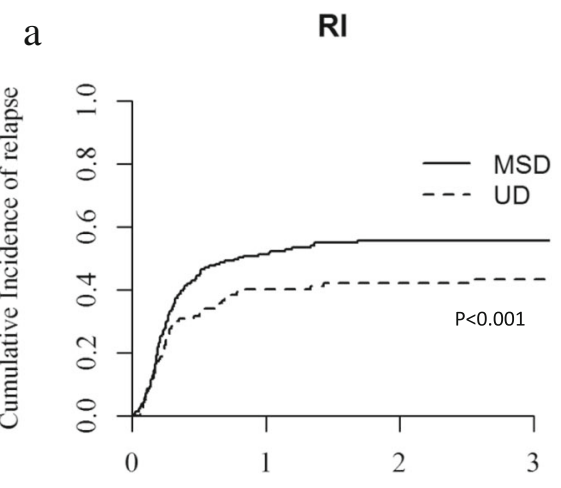

Years after transplantation

C

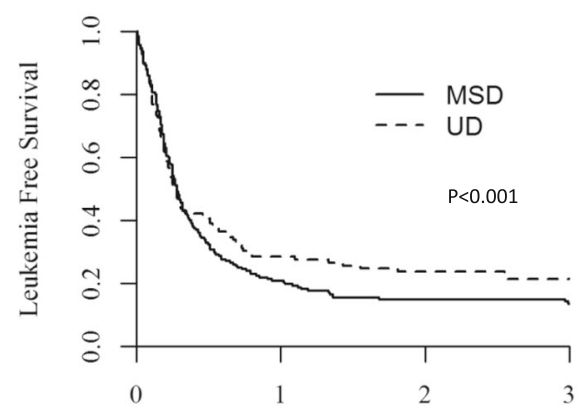

Years after transplantation

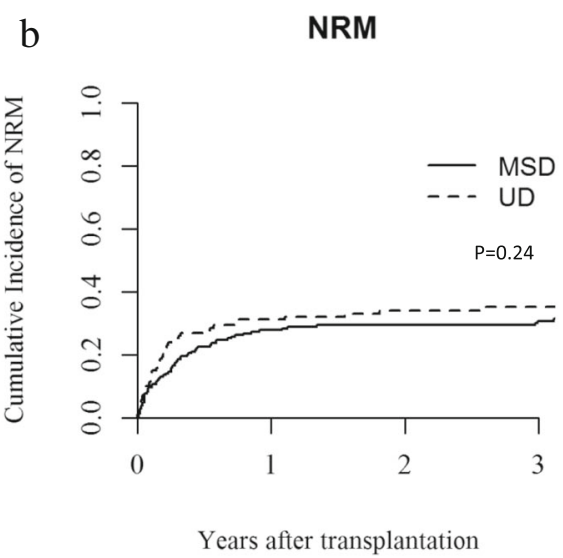

d os

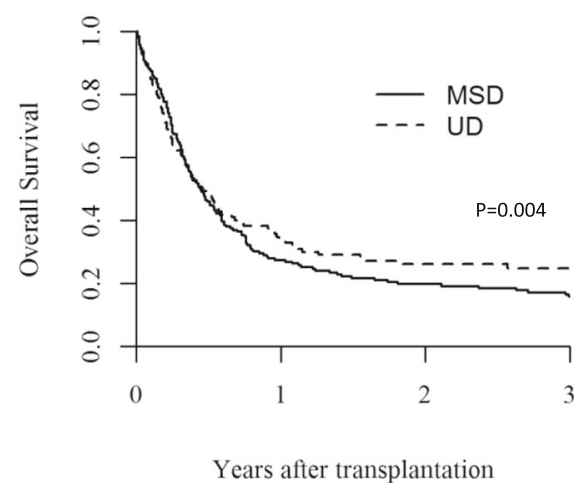

Fig. 1 a Relapse incidence. b Non-relapse mortality. c Leukemia-free survival. d Overall survival by type of donor

$1.18-2.48 ; p<0.001)$. Causes of death are reported in Table 3.

\section{LFS and OS}

With a median follow-up of 2.4 (range $0.3-13$ ) years, the 2 years probability of LFS was 21 vs $26 \%(p<0.001)$ and OS was 26 vs $33 \%(p<0.004)$ for MSD and UD recipients, respectively (Fig. 1c, d). In adjusted multivariate analysis (Table 2), LFS was higher for patients transplanted with UD $(\mathrm{HR}=0.83,95 \% \mathrm{CI} 0.72-0.96 ; p<0.01)$ and for those with a shorter interval between diagnosis and HSCT $(\mathrm{HR}=0.67$, $95 \%$ CI $0.59-0.77 ; p<0.001)$. Chronic GVHD as timedependent variable was associated with higher OS $(\mathrm{HR}=$ $0.69,95 \%$ CI $0.56-0.84 ; p<0.001)$, and no differences were observed for LFS (HR $=0.99,95 \%$ CI $0.80-1.21 ; p=0.90$ ).

\section{Outcomes according to HLA-match}

We performed a subgroup analysis to evaluate outcomes according to HLA disparity (UD 10/10 or 9/10 as compared to MSD). There were no differences for neutrophil engraftment ( 92 vs 89 vs $93 \%, p=0.20$ ), chronic GVHD (25\% in all groups, $p=0.96)$, and NRM
( 23 vs 23 vs $30 \%, p=0.26$ ) between the three groups. CI of relapse was higher for MSD as compared to $10 / 10$ or $9 / 10$ UD ( 57 vs 50 vs $45 \%, p=0.0003$ ). Patients in the MSD group had lower LFS (20\% in MSD vs $27 \%$ in $10 / 10$ and $25 \%$ in $9 / 10$ UD, $p=0.003$ ), and lower OS (26\% in MSD vs $34 \%$ in $10 / 10$ and $29 \%$ in $9 / 10 \mathrm{UD}, p=0.01$ ). No differences were found in the multivariate analysis (Table 4) for MSD versus 9/10 UD for RI ( $\mathrm{HR}=0.77,95 \% \mathrm{CI} 0.57-1.05$; $p=0.10)$, NRM ( $\mathrm{HR}=1.32,95 \%$ CI $0.87-2.00 ; p=0.19$ ), LFS (HR $=0.92$, $95 \%$ CI $0.72-1.18 ; p=0.53)$, and OS $(\mathrm{HR}=1.00,95 \%$ CI $0.78-1.29 ; p=0.99)$.

\section{Discussion}

AML recurrence still represents one of the most difficult scenarios associated with poor prognosis. Most patients often do not respond to reinduction therapies which are also hampered by toxicities. This might leave patients unfit for additional therapies. On the other hand, HSCT is a treatment strategy that may offer possibility of cure, although survival does not exceed 20 to $35 \%$ at 4 years $[11,12]$. The increased availability of donors, the use of non-myeloablative or RIC regimens, and the progress in 
Table 2 Multivariate analysis for patients with AML in primary relapse

\begin{tabular}{|c|c|c|}
\hline Variable & HR $(95 \% \mathrm{Cl})$ & $p$ \\
\hline \multicolumn{3}{|l|}{$\mathrm{Rl}$} \\
\hline UD vs MSD & $0.76(0.64-0.89)$ & $<0.01$ \\
\hline $\mathrm{Age}^{\mathrm{a}}$ & $0.99(0.99-1.00)$ & 0.02 \\
\hline Time from $d x$ to $T x>9$ months & $0.62(0.53-0.72)$ & $<0.01$ \\
\hline Female donor to male recipient & $0.91(0.74-1.11)$ & 0.35 \\
\hline SAML & $0.99(0.79-1.23)$ & 0.92 \\
\hline previous auto-HSCT & $1.22(0.86-1.73)$ & 0.26 \\
\hline Positive recipient CMV serology & $0.96(0.80-1.15)$ & 0.68 \\
\hline Positive donor CMV serology & $1.03(0.86-1.22)$ & 0.77 \\
\hline RIC & $1.21(1.02-1.42)$ & 0.03 \\
\hline \multicolumn{3}{|l|}{ NRM } \\
\hline UD vs MSD & $1.04(0.80-1.35)$ & 0.79 \\
\hline $\mathrm{Age}^{\mathrm{a}}$ & $1.02(1.01-1.03)$ & $<0.01$ \\
\hline Time from $d x$ to $T x>9$ months & $0.82(0.64-1.06)$ & 0.12 \\
\hline Female donor to male recipient & $0.86(0.62-1.21)$ & 0.40 \\
\hline SAML & $1.39(1.03-1.87)$ & 0.03 \\
\hline previous auto-HSCT & $1.49(0.91-2.43)$ & 0.11 \\
\hline Positive recipient CMV serology & $0.98(0.74-1.30)$ & 0.89 \\
\hline Positive donor CMV serology & $0.98(0.74-1.28)$ & 0.86 \\
\hline RIC & $0.96(0.74-1.24)$ & 0.74 \\
\hline \multicolumn{3}{|l|}{ LFS } \\
\hline UD vs MSD & $0.83(0.72-0.96)$ & 0.01 \\
\hline $\mathrm{Age}^{\mathrm{a}}$ & $1.00(0.99-1.00)$ & 0.82 \\
\hline Time from $d x$ to $T x>9$ months & $0.67(0.59-0.77)$ & $<0.01$ \\
\hline Female donor to male recipient & $0.90(0.75-1.07)$ & 0.22 \\
\hline SAML & $1.11(0.93-1.32)$ & 0.26 \\
\hline previous auto-HSCT & $1.29(0.97-1.71)$ & 0.08 \\
\hline Positive recipient CMV serology & $0.97(0.84-1.13)$ & 0.72 \\
\hline Positive donor CMV serology & $1.01(0.87-1.17)$ & 0.87 \\
\hline $\mathrm{RIC}$ & $1.13(0.98-1.30)$ & 0.09 \\
\hline \multicolumn{3}{|l|}{ OS } \\
\hline UD vs MSD & $0.89(0.77-1.03)$ & 0.12 \\
\hline $\mathrm{Age}^{\mathrm{a}}$ & $1.00(1.00-1.01)$ & 0.36 \\
\hline Time from $d x$ to $T x>9$ months & $0.71(0.61-0.81)$ & $<0.01$ \\
\hline Female donor to male recipient & $0.86(0.72-1.03)$ & 0.10 \\
\hline SAML & $1.09(0.91-1.31)$ & 0.34 \\
\hline previous auto-HSCT & $1.25(0.93-1.68)$ & 0.15 \\
\hline Positive recipient CMV serology & $0.96(0.82-1.12)$ & 0.61 \\
\hline Positive donor CMV serology & $0.99(0.85-1.16)$ & 0.92 \\
\hline $\mathrm{RIC}$ & $1.01(0.88-1.17)$ & 0.88 \\
\hline
\end{tabular}

Abbreviations: $H R$ hazard ratio, $C l$ confidence interval, $R I$ relapse incidence, NRM non-relapse mortality, LFS leukemiafree survival, OS overall survival, UD unrelated donor, MSD matched sibling donor, cont continuous, $d x$ diagnosis, tx transplant, sAML secondary acute myeloid leukemia, auto-HSCT autologous hematopoietic stem cell transplantation, CMV cytomegalovirus, $R I C$ reduced-intensity conditioning regimen

${ }^{\mathrm{a}}$ As continuous variable
Table 3 Causes of death for patients with MSD and UD

\begin{tabular}{lll}
\hline & MSD & UD \\
\hline Cardiac toxicity & $3(0.4 \%)$ & $3(0.8 \%)$ \\
Hemorrhage & $12(1.7 \%)$ & $5(1.3 \%)$ \\
Failure/Rejection & $3(0.4 \%)$ & $1(0.3 \%)$ \\
Veno Occlusive Disease & $11(1.6 \%)$ & $14(3.7 \%)$ \\
Infection & $105(15.1 \%)$ & $64(16.8 \%)$ \\
Interstitial Pneumonia & $11(1.6 \%)$ & $7(1.8 \%)$ \\
Graft versus host disease & $72(10.4 \%)$ & $50(13.1 \%)$ \\
Original disease & $436(62.3 \%)$ & $222(58.1 \%)$ \\
Secondary malignancy & $2(0.3 \%)$ & $0(0 \%)$ \\
Other transplant related & $32(4.6 \%)$ & $9(2.4 \%)$ \\
Multi Organ Failure & $8(1.2 \%)$ & $7(1.8 \%)$ \\
Unknown & 14 & 5 \\
\hline
\end{tabular}

supportive care, translating in survival improvements, made HSCT available and possible in almost all patients. Therefore, a rapid donor search should be promptly initiated in high-risk AML patients [4].

In the present study, we compared the outcomes of patients undergoing allogeneic HSCT for AML in primary relapse either from a MSD or from a matched or single-allele mismatched UD.

Several retrospective studies on patients transplanted with active disease have been reported. The French Society of Bone Marrow Transplantation (SFGM) reported outcomes of 379 patients transplanted for high-risk AML with a 5-year probability of LFS approximately of $10 \%$. In multivariate analysis the use of a matched or mismatched UD was associated with lower OS [13].

Few retrospective studies or case series limited to a small subset of highly selected patients receiving a MAC HSCT for active AML have been reported in the literature [13, 14]: a large CIBMTR study in AML in relapse or primary induction failure showed a 3-year OS of $19 \%$.

The German Transplant Study in 113 patients undergoing a RIC transplant showed a probability of LFS of $49 \%$ for patients with less than $5 \%$ bone marrow blasts and $14 \%$ for those with more than $20 \%$ marrow blasts at time of HSCT [15]. Moreover, patients transplanted from a MSD had better event-free survival as compared to patients transplanted from UD. A phase II study from van Besien et al. in patients with AML or myelodysplastic syndrome included 28 patients with active AML undergoing a RIC transplant with fludarabine, melphalan, and alemtuzumab, with a 1-year LFS of $25 \%$. No differences were found in the whole population outcomes according to the type of donor [16].

In our cohort, we observed that patients undergoing a RIC had a higher risk of relapse when compared to MAC transplant, in accordance with previous reports $[17,18]$. 
Table 4 Multivariate analysis for patients with AML in primary relapse according to HLA mismatch

\begin{tabular}{|c|c|c|}
\hline Variable & $\mathrm{HR}(95 \% \mathrm{Cl})$ & $p$ \\
\hline \multicolumn{3}{|l|}{ Rl } \\
\hline UD 10/10 & $0.75(0.63-0.90)$ & $<0.01$ \\
\hline UD $9 / 10$ & $0.77(0.57-1.05)$ & 0.10 \\
\hline $\mathrm{Age}^{\mathrm{a}}$ & $0.99(0.99-1.00)$ & 0.02 \\
\hline Time from $d x$ to $T x>9$ mo & $0.62(0.52-0.72)$ & $<0.01$ \\
\hline Female donor to male recipient & $0.91(0.74-1.11)$ & 0.34 \\
\hline SAML & $0.99(0.79-1.23)$ & 0.92 \\
\hline previous auto-HSCT & $1.22(0.86-1.73)$ & 0.26 \\
\hline Positive recipient CMV serology & $0.96(0.80-1.15)$ & 0.68 \\
\hline Positive donor CMV serology & $1.03(0.86-1.22)$ & 0.77 \\
\hline $\mathrm{RIC}$ & $1.21(1.02-1.42)$ & 0.03 \\
\hline \multicolumn{3}{|l|}{ NRM } \\
\hline UD 10/10 & $0.97(0.74-1.29)$ & 0.86 \\
\hline UD 9/10 & $1.32(0.87-2.00)$ & 0.19 \\
\hline $\mathrm{Age}^{\mathrm{a}}$ & $1.02(1.01-1.03)$ & $<0.01$ \\
\hline Time from $d x$ to $T x>9$ mo & $0.81(0.62-1.04)$ & 0.10 \\
\hline Female donor to male recipient & $0.86(0.61-1.20)$ & 0.38 \\
\hline SAML & $1.40(1.04-1.90)$ & 0.03 \\
\hline previous auto-HSCT & $1.50(0.92-2.45)$ & 0.10 \\
\hline Positive recipient CMV serology & $0.96(0.72-1.28)$ & 0.80 \\
\hline Positive donor CMV serology & $0.98(0.75-1.29)$ & 0.90 \\
\hline $\mathrm{RIC}$ & $0.96(0.74-1.25)$ & 0.78 \\
\hline \multicolumn{3}{|l|}{ LFS } \\
\hline UD 10/10 & $0.81(0.70-0.94)$ & 0.01 \\
\hline UD 9/10 & $0.92(0.72-1.18)$ & 0.53 \\
\hline $\mathrm{Age}^{\mathrm{a}}$ & $1.00(0.99-1.00)$ & 0.82 \\
\hline Time from $\mathrm{dx}$ to $T x>9 \mathrm{mo}$ & $0.67(0.58-0.76)$ & $<0.01$ \\
\hline Female donor to male recipient & $0.89(0.75-1.07)$ & 0.21 \\
\hline SAML & $1.11(0.93-1.33)$ & 0.24 \\
\hline previous auto-HSCT & $1.30(0.98-1.72)$ & 0.07 \\
\hline Positive recipient CMV serology & $0.97(0.83-1.13)$ & 0.67 \\
\hline Positive donor CMV serology & $1.01(0.88-1.17)$ & 0.86 \\
\hline $\mathrm{RIC}$ & $1.13(0.98-1.30)$ & 0.08 \\
\hline \multicolumn{3}{|l|}{ OS } \\
\hline UD 10/10 & $0.87(0.74-1.01)$ & 0.07 \\
\hline UD 9/10 & $1.00(0.78-1.29)$ & 0.99 \\
\hline $\mathrm{Age}^{\mathrm{a}}$ & $1.00(1.00-1.01)$ & 0.36 \\
\hline Time from $d x$ to $T x>9 \mathrm{mo}$ & $0.70(0.61-0.81)$ & $<0.01$ \\
\hline Female donor to male recipient & $0.85(0.71-1.03)$ & 0.09 \\
\hline SAML & $1.10(0.91-1.32)$ & 0.31 \\
\hline previous auto-HSCT & $1.25(0.93-1.69)$ & 0.14 \\
\hline
\end{tabular}

Table 4 Multivariate analysis for patients with AML in primary relapse according to HLA mismatch (Continued)

\begin{tabular}{llc}
\hline Positive recipient CMV serology & $0.95(0.81-1.12)$ & 0.56 \\
Positive donor CMV serology & $0.99(0.85-1.16)$ & 0.94 \\
RIC & $1.01(0.88-1.17)$ & 0.86 \\
\hline Abbreviations: HR hazard ratio, Cl confidence interval, $R$ r relapse incidence, NRM \\
non-relapse mortality, LFS leukemia-free survival, OS overall survival, UD unrelated \\
donor, MSD matched sibling donor, cont continuous, dx diagnosis, $t x$ transplant, \\
sAML secondary acute myeloid leukemia, auto-HSCT autologous hematopoietic \\
stem cell transplantation, CMV cytomegalovirus, RIC reduced-intensity \\
conditioning regimen \\
aAs continuous variable
\end{tabular}

Some of the patients in the current study received a sequential regimen: however, numbers were too small to analyze the impact of this regimen on HSCT outcomes.

Compared to MSD recipients, in our series patients transplanted with UD experienced higher OS, LFS, and lower RI. This was confirmed in the multivariate analysis, where the type of donor was an independent risk factor for LFS and relapse incidence. This could be related to a stronger graft versus leukemia (GVL) effect in the UD group due to a higher likelihood of mismatches in minor histocompatibility antigens [19].

Increase in GVHD in case of UD is reported both in the MAC and RIC setting, with a correlation between severity of GVHD and HLA mismatch (29). Importantly in our series, acute and chronic GVHD were not different either according to the type of donor or to HLA mismatches. However, given the retrospective nature of our study, we were not able to evaluate the effect of the single-allele mismatch and the HLA locus for addressing the GVL effect.

In the UD setting, a lower incidence of relapse in patients experiencing chronic GVHD has been reported [20]. Of note, in our series, in accordance with previous reports [21], chronic GVHD as a time-dependent variable was associated with lower risk of RI and higher OS.

Similar results were found by Lee et al. who reported a better disease control in extensive as compared to limited chronic GVHD, this being counterbalanced by morbidity from chronic GVHD [22].

When focusing on conditioning intensity, Weisdorf et al. reported a longer GVL benefit in patients experiencing GVHD after a RIC transplant as compared to patients undergoing a MAC transplant [23]. This study highlights the importance of immune modulation, especially in the context of RIC transplant.

Importantly, improvements in supportive care after HSCT over the years, including the advent of new antifungal and antiviral drugs, allowed better survival and reduction of NRM, with a wider number of patients eligible for post-transplant immune modulation with better disease control. 
One may argue that patients receiving RIC may have benefit of earlier withdrawal of immunosuppressive treatment or post HSCT maintenance therapy, or donor lymphocyte infusion and therefore without differences in LFS. However, we did not investigate the type of post HSCT strategy, being related to the different transplant policy.

We confirm that the interval from diagnosis to HSCT was one of the factors associated with relapse, LFS, and OS, independently from the type of donor, highlighting the need to rapidly look for a donor and perform HSCT early to ensure long-term disease control. The place of unmanipulated haploidentical donor transplant need to be considered in this setting to establish the adapted algorithm for donor selection in this setting of patients.

Our study has some limitations as a retrospective registry analysis. Although patients were categorized by their disease biology and the multivariate modeling may have adjusted for various patient-, disease-, and transplantationrelated factors, there may be factors of importance that we have not been able to take into account, such as the reason why those patients were not transplanted in first CR.

Further studies are needed to highlight the place of sequential approach in such a high-risk patients with otherwise limited options, as reported also using other regimen combination [24], and other post-transplant strategies such as early withdrawal of immunosuppressive treatment and immunomodulating agents or preemptive use of donor lymphocyte infusions should be considered early in this high-risk population to enhance long-term disease control.

\section{Conclusions}

Our data suggest that the use of UD is feasible and effective in patients with AML undergoing allogeneic HSCT in primary relapse, with even better outcomes in terms of LFS, RI and OS in patients transplanted from MSD. The role of one antigen mismatch UD in this setting should be evaluated in a larger and more homogenous series. Moreover, as timing to HSCT still remains one of the most important factors influencing transplant outcomes, it is important to promptly start donor search on the registries when a MSD is not available, in order to identify a suitable UD. Our data confirm that a strong disease control is possible with a single-allele mismatched UD with no increased toxicity. In patients not eligible for a full intensity regimen, use of RIC regimens, might be a valid option to improve disease control and survival. Despite better results for patients given UD, outcome of patients transplanted in primary relapse is still not satisfactory. Further prospective studies investigating the implementation of posttransplant immunomodulating strategies are warranted in this high-risk subset of patients.
Acknowledgements

The authors thank Emmanuelle Polge from the office of the ALWP of EBMT.

Funding

The study was supported in part by the grants of Ministry of Health CR 15-30661A, 15-25809A, by the grant TA ČR TA04010727.

Availability of data and materials

The dataset supporting the conclusions of this article are available in the ALWP of EBMT in Paris, 184 rue Faubourg Saint Antoine.

Authors' contributions

$A R, M L$, and $A N$ designed the study. $A R$ and $M L$ performed the statistical analysis. $A R$ and $G B$ wrote the manuscript. GE, DB, JT, AG, RS, BG, JF, MM, MS, PJ, RA, NK, and MM provided cases for the study. All authors edited and approved the manuscript.

\section{Competing interests}

The authors declare that they have no competing interests.

Consent for publication

Not applicable

Ethics approval and consent to participate

The scientific boards of the ALWP of EBMT approved this study. All patients gave written informed consent for the use of their data.

\section{Consent to publish}

Not applicable for individual patient data. This is a pooled analysis.

\section{Author details}

'Service d'Hématologie et Thérapie Cellulaire, Hôpital Saint Antoine, AP-HP, 184 Rue du Faubourg Saint Antoine, 75012 Paris, France. ${ }^{2}$ Department of Hematology and Marrow Transplantation, University Federico II of Naples, Naples, Italy. ${ }^{3}$ Medical Clinic and Polyclinic, University Hospital Carl Gustav Carus, Technical University Dresden, Dresden, Germany. ${ }^{4}$ Department of Bone Marrow Transplantation, University Hospital Essen, Essen, Germany. ${ }^{5}$ Department of Internal Medicine III, UH of Munich (LMU), Munich, Germany. ${ }^{6}$ Department of Hematology, Hemostasis, Oncology, and Stem Cell Transplantation, Hannover Medical School, Hannover, Germany. 'DDepartment of Haematology, Oncology Helios-Klinikum Berlin-Buch, Schwanebecker Chaussee 50, 13125 Berlin, Germany. ${ }^{8}$ Department of Hematology, Oncology and Stem Cell Transplantation, Asklepios Hospital St. Georg, Hamburg, Germany. ${ }^{9}$ Department of Hematology/Oncology and Stem Cell Transplantation, University Medical Center, Freiburg, Germany. ${ }^{10}$ Department of Hematology, Lyon-Sud Hospital, Hospices Civils de Lyon, Pierre Bénite, France. ${ }^{11}$ Department of Medicine A, University of Muenster, Munster, Germany. ${ }^{12}$ Departments of Hematology/Oncology, Charles University Hospital, Pilsen, Czech Republic. ${ }^{13}$ Charité Universitatsmedizin, 10117 Berlin, Germany. ${ }^{14}$ University Medical Center Hamburg-Eppendorf, Hamburg, Germany. ${ }^{15}$ Hôpital Saint-Antoine, Paris University UPMC, INSERM U938, Paris, France. ${ }^{16}$ Université Pierre and Marie Curie, Paris, France. ${ }^{17}$ Hematology Division, Chaim Sheba Medical Center, Tel Hashomer, Israel. ${ }^{18}$ ALWP Office, Hôpital Saint Antoine, AP-HP, Paris, France.

Received: 19 July 2016 Accepted: 8 September 2016

Published online: 17 September 2016

\section{References}

1. Burnett AK. Acute myeloid leukemia: treatment of adults under 60 years. Rev Clin Exp Hematol. 2002;6:26-45.

2. Nagler A, Savani B, Labopin M, et al. Outcomes after use of two standard ablative regimens in patients with refractory acute myeloid leukaemia: a retrospective, multicentre, registry analysis. Lancet Haematol. 2015;2:e384-92.

3. McSweeney PA, Niederwieser D, Shizuru JA, et al. Hematopoietic cell transplantation in older patients with hematologic malignancies: replacing high-dose cytotoxic therapy with graft-versus-tumor effects. Blood. 2001;97:3390-400.

4. Gragert L, Eapen M, Williams E, et al. HLA match likelihoods for hematopoietic stem-cell grafts in the U.S. registry. N Engl J Med. 2014;371:339-48. 
5. Yakoub-agha I, Mesnil F, Kuentz M, et al. Allogeneic marrow stem-cell transplantation from human leukocyte antigen -identical siblings versus human leukocyte antigen-allelic-matched unrelated donors (10 / 10) in patients with standard-risk hematologic malignancy: a prospective study from the French Society of Bone Marrow Transplantation and Cell Therapy. J Clin Oncol. 2006;24:5695-702.

6. Grimwade D, Hills RK, Moorman AV, et al. Refinement of cytogenetic classification in acute myeloid leukaemia: Determination of prognostic significance of rarer recurring chromosomal abnormalities amongst 5,876 younger adult patients treated in the UK Medical Research Council trials. Blood. 2010;116:354-65.

7. Shulman H, Sullivan KM, Weiden PL, et al. Chronic graft-versus-host syndrome in man. A long-term clinicopathologic study of 20 Seattle patients. Am J Med. 1980;69:204-17.

8. Kaplan EL, Meier P. Nonparametric estimation from incomplete observations. J Am Stat Assoc. 1958:53:457-81.

9. Fine JP, Gray R. A proportional hazards model for the subdistribution of a competing risk. J Am Stat Assoc. 1999;94:496-509.

10. Schmid C, Schleuning M, Ledderose G, et al. Sequential regimen of chemotherapy, reduced-intensity conditioning for allogeneic stem-cell transplantation, and prophylactic donor lymphocyte transfusion in high-risk acute myeloid leukemia and myelodysplastic syndrome. J Clin Oncol. 2005;23:5675-87.

11. Duval M, Klein JP, He W, et al. Hematopoietic stem-cell transplantation for acute leukemia in relapse or primary induction failure. J Clin Oncol. 2010;28:3730-8

12. Schlenk RF, Dohner K, Mack S, et al. Prospective evaluation of allogeneic hematopoietic stem-cell transplantation from matched related and matched unrelated donors in younger adults with high-risk acute myeloid leukemia: German-Austrian Trial AMLHD98A. J Clin Oncol. 2010;28:4642-8.

13. Michallet $M$, Thomas $X$, Vernant JP, et al. Long-term outcome after allogeneic hematopoietic stem cell transplantation for advanced stage acute myeloblastic leukemia: a retrospective study of 379 patients reported to the Societé Française de Greffe de Moelle. Bone Marrow Transplant. 2000;26:1157-63.

14. Wong R, Shahjahan M, Wang X, et al. Prognostic factors for outcomes of patients with refractory or relapsed acute myelogenous leukemia or myelodysplastic syndromes undergoing allogeneic progenitor cell transplantation. Biol Blood Marrow Transplant. 2005;11:108-14.

15. Sayer HG, Kröger M, Beyer J, et al. Reduced intensity conditioning for allogeneic hematopoietic stem cell transplantation in patients with acute myeloid leukemia: disease status by marrow blasts is the strongest prognostic factor. Bone Marrow Transplant. 2003;31:1089-95.

16. van Besien K, Artz A, Smith S, et al. Fludarabine, melphalan, and alemtuzumab conditioning in adults with standard-risk advanced acute myeloid leukemia and myelodysplastic syndrome. J Clin Oncol. 2005;23:5728-38.

17. Aoudjhane M, Labopin M, Gorin NC, et al. Comparative outcome of reduced intensity and myeloablative conditioning regimen in HLA identical sibling allogeneic haematopoietic stem cell transplantation for patients older than 50 years of age with acute myeloblastic leukaemia: a retrospective survey from the Acute Leukemia Working Party (ALWP) of the European group for Blood and Marrow Transplantation (EBMT). Leukemia. 2005;19:2304-12.

18. Alyea EP, Kim HT, Ho V, et al. Comparative outcome of nonmyeloablative and myeloablative allogeneic hematopoietic cell transplantation for patients older than 50 years of age. Blood. 2005;105:1810-4.

19. Shaw BE, Gooley TA, Malkki M, et al. The importance of HLA-DPB1 in unrelated donor hematopoietic cell transplantation. Blood. 2007;1 10:4560-7.

20. Remberger M, Mattsson J, Hentschke $P$, et al. The graft-versus-leukaemia effect in haematopoietic stem cell transplantation using unrelated donors. Bone Marrow Transpl. 2002;30:761-8.

21. Ringden O, Labopin M, Gluckman E, et al. Strong antileukemic effect of chronic graft-versus-host disease. Transplant Proc. 1997;29:733-4.

22. Lee SJ, Klein JP, Barrett AJ, et al. Severity of chronic graft-versus-host disease: association with treatment-related mortality and relapse. Blood. 2002;100:406-14.

23. Weisdorf D, Zhang MJ, Arora M, et al. Graft-versus-host disease induced graftversus-leukemia effect: greater impact on relapse and disease-free survival after reduced intensity conditioning. Biol Blood Marrow Transplant. 2012;18:1727-33.

24. Chevallier P, Labopin M, Socié G, et al. Results from a clofarabine-busulfancontaining, reduced-toxicity conditioning regimen prior to allogeneic stem cell transplantation: the phase 2 prospective CLORIC trial. Haematologica. 2014;99:1486-91.

\section{Submit your next manuscript to BioMed Central and we will help you at every step:}

- We accept pre-submission inquiries

- Our selector tool helps you to find the most relevant journal

- We provide round the clock customer support

- Convenient online submission

- Thorough peer review

- Inclusion in PubMed and all major indexing services

- Maximum visibility for your research

Submit your manuscript at www.biomedcentral.com/submit
Biomed Central 